\title{
Joint Optimization in Condition-Based Maintenance and Inventory Policy for the Repairable System
}

 \\ Department of Equipment Command and Management, Army Engineering University of PLA, Shijiazhuang 050003, China \\ Correspondence should be addressed to Quan Shi; duke970618@aliyun.com
}

Received 6 July 2021; Revised 3 August 2021; Accepted 18 August 2021; Published 31 August 2021

Academic Editor: Kauko Leiviskä

Copyright ( 92021 Huayang Deng et al. This is an open access article distributed under the Creative Commons Attribution License, which permits unrestricted use, distribution, and reproduction in any medium, provided the original work is properly cited.

\begin{abstract}
In the modern industry, to reduce support pressure, a variety of suppliers have begun to use the same kind of units in many types of equipment. However, highly integrated equipment is more difficult to use in traditional reliability-centered maintenance (RCM) models, and researchers start steering based on the state monitoring method. This paper mainly discusses the remaining useful life (RUL) prediction method based on the Wiener process and introduces the improved condition-based maintenance policy. Combined with the $(S-1, S)$ policy, the joint policy and the model considering the repairable policy are built for the multiunit system. Finally, the optional decision combination is obtained. Except that, the advantage of the joint optimization in a multiunit system and the characteristics of repairable policy in the joint model are analyzed.
\end{abstract}

\section{Introduction}

In industrial, military, and other fields, many customers purchase equipment from the same supplier for convenience. One of the important reasons is that the suppliers begin to use identical units in their equipment, especially nonstandard units. It is good for equipment upgrades and customer protection. However, with the emergence of high precision, high information, and highly integrated equipment, the traditional fault prediction based on RCM using classical fault law experience shows some limitations in the more complex systematic analysis [1]. With the development of sensor technology, many researchers begin to establish equipment life degradation models based on the data-driven analysis, so as to predict the RUL more accurately [2].

The main data-driven methods used in prediction are the Gamma process, the Gaussian inverse process, and the Wiener process. After the exploration of many researchers, the data-driven methods can be adapted to more and more modeling environments and used flexibly by adjusting formula parameters and expanding formula [3]. So far, it has been widely used in the field of reliability [4-6]. Compared with the characteristics of the three models, the Gamma process and the Gaussian inverse process are only suitable for modeling monotone degradation curves. In contrast, the Wiener process is more suitable for nonmonotonic degradation processes caused by repair, self-healing, or use intensity [7]. It also plays an important prerequisite of choosing a proper inventory policy [8].

In the research of inventory policy, the application is very high [9]. As two kinds of the most basic and classical ones, they are still widely used in a modern supply chain like the $(T, S)$ and the $(s, S)$ policy. In the $(T, S)$ policy, the inventory will replenish to maximum inventory $S$ after a check cycle $T$ goes. As for the $(s, S)$ policy, when the inventory decreases to the minimum inventory $s$, it will be resupplied to maximum inventory $S$. Condition-based maintenance $(\mathrm{CBM})$ is one of the rather complicated preventive maintenance policies. The $(s, S)$ policy that pays more attention to the change of inventory is closer along with the characteristics of CBM, which is based on the state of parts [10]. The $(S-1, S)$ policy is one of the improved $(s, S)$ policies [11]. After a unit is replaced, the resupply applicant will be sent. It is suitable for the multiunit system, which is discussed in the former paragraph.

This article introduces the joint optimization problem of condition-based maintenance and inventory policy. The proposed joint policy is built for the repairable multiunit 
system; the mathematical model combines the improved condition-based maintenance policy based on the RUL prediction through the Wiener process simulation and the $(S-1, S)$ policy innovatively. A case is given. With the optimization of the PSO algorithm, the optional decision combination is obtained. Meanwhile, this article investigates the advantage of the $(S-1, S)$ policy in the multiunit system and the characteristics of the repairable policy. This joint policy in this paper is built for the valuable parts and can be applied to the spare parts ordering in more scenarios.

The rest of this paper is organized as follows: Section 2 is the literature review of the joint optimization of CBM and inventory policy and repairable policy. The main assumptions and notations in this article are discussed in Section 3. According to the conditions and constraints, Section 4 mainly introduces the joint policy of CBM and inventory policy for the multiple identical units and the modeling process. Except that, the model of multiperiod resupply and repairable policy are also mentioned. In Section 5, a case analysis will be given and adopted by the Monte Carlo simulation and the particle swarm optimization (PSO) algorithm to optimize the joint decision combinations that conclude the check cycle, preventive threshold, and maximum inventory. Besides, the advantages of joint optimization for the multiunit system and analysis in the repairable policy are also shown. Conclusions and future work are given in Section 6.

\section{Literature Review}

2.1. Residual Life Prediction Based on the Wiener Process. The traditional RCM analysis mainly depends on the calculation results of the consumption statistics of units. However, their performance degradation processes under different environments and conditions are different. Therefore, the performance degradation model and analysis of a specific system through the data-driven method based on state monitoring can more accurately reflect the fault law of the system in the real situation. After obtaining the status data, there are two main ways to use it. One is used to analyze the degradation law after directly processing. The other is used to predict the next failure time in order to take corresponding maintenance measures in time through the prediction technology. This time difference between the time of obtaining data and after prediction to fail is called residual life (RUL).

The Wiener process is one of the commonly used methods for modeling performance degradation in reliability assessment based on the data-driven method [7]. The Wiener process is a random function, driven by Brownian motion (BM). It was usually used to establish the linear model in the beginning. In the current research, it has gradually developed to general and nonlinear problems related to time or state [12]. Wang et al. ort out the diversity of model research, including time diversity, unit diversity, measurement diversity, and structural diversity [13]. Another point of the study is to consider the impact of the working environment on the performance degradation process. Bian and Gebraeel add covariates in the Wiener process as the environmental factors in the formula [14]. The model based on the Wiener process has also been applied to the field of spare parts. Elwany and Gebraeel firstly combined the maintenance decision based on the Wiener process with the spare parts ordering to evaluate the optimal replacement and ordering time. They finally put forward the general framework of the joint policy as well [15]. After that, Zhang and Chen et al. tried to introduce the Bayesian model into the joint decision model and update the model parameters with real-time performance data [16]. On the other hand, Zhang and Zeng extended the decision-making process to propose a random order lead time for the inventory [17].

2.2. Inventory Policy. The maintenance policy leads to the inventory policy [18]. Talking about all inventory policies, according to the optimization goal, they can be divided into two main aspects. One is mainly represented by the inventory policies whose research goal is the check cycle $(T)$, such as the $(T, S)$ and $(T, Q)$ policies [19]. However, in the long-term research, experts found that it has limitations in applying due to the uncertainty of actual demand [20]. Therefore, the rigid and fixed inventory policies are difficult to meet the needs of multiple maintenance policies. Researchers began to discuss other kinds of inventory policy represented mainly by the $(s, S)$ and $(s, Q)$ policies $[10,20,21]$.

The corresponding situation of units leads to the change of inventory. In this view, the inventory node is the center of optimization. However, due to the high importance of units using the data-driven method, the units' working time is rather long. The probability of failure in a short time is small from start to work. A large number of spare parts are piled up in the warehouse, which will result in a substantial increase in the proportion of inventory cost to the total cost. Lots of spare parts in some smaller warehouses cannot be transferred. Furthermore, there could be a large number of faulty spare parts and losses resulting from it. On the contrary, in the multiunit system, it is highly likely that multiple units will fail at the same time. For this reason, there is going to be a great consumption of spare parts. Considering the developed Internet and logistics industry, the researchers put forward the $(S-1, S)$ policy. This policy aims to resupply spare parts as soon as possible. After a unit is replaced, the resupply applicant will be sent out. Sherbrooke firstly developed the policy into the METRIC model under the limited cost and minimum out-of-stock [22]. After that, Sherbrooke developed the model to the VARI-METRIC model based on the former model [23]. Grave proposed a simple algorithm for this research, which can be used in the calculation [24].

2.3. Joint Optimization. There are lots of research studies on the joint policy. Acharya studied the joint optimization of preventive maintenance and inventory policy in 1984 [25]. Many researchers tried to combine different maintenance policies and inventory policies. The maintenance policies conclude age-based replacement [26], block replacement 
[27], planned maintenance [28], and CBM. Van Horenbeek et al. summarized the joint policy of maintenance and inventory in many aspects concluding inventory policy, maintenance policy, resupply network, single-unit system, multiunit system, and so on [29]. The previous literature focuses on traditional failure laws, such as exponential distribution and Poisson distribution [28].

In recent years, data-driven fault analysis was gradually developed, especially the Wiener process. Wang et al. [30] built the joint model in the CBM and inventory policy for a single-unit system. Wang et al. [31] established the joint policy concluding the maintenance and inventory policies for the unrepairable unit. In this policy, the different conditions about the replacement of units were considered carefully in order to construct the objective function for the minimum expected cost per unit time. Finally, the optional maintenance threshold and maximum inventory were obtained by the RUL prediction based on the historical data. But there is a regret that the system check cycle is not optimized. In reference [32], the researchers analyzed the sensitivity of the check cycle in the result analysis. However, check cycle is not included in the joint optimization calculation as one of the optimization objects. Above all, the object was limited in the single-unit system among the former references. Zheng et al. [33] built the joint policy of the CBM and $(S-1, S)$ policy for a single unit. In reference [33], the advantage of the $(S-1, S)$ policy for single-unit optimization has been verified tentatively. Wang et al. [34] began to extend the joint optimization to multiple units. However, resulting from the complexity of the model and simulation, the joint model has adopted the Markov chain to analyze, not the method of RUL prediction. Wang et al. [35] built the joint policy of CBM and inventory for a multiunit system. A simulation model was developed for the joint policy through Monte Carlo simulation in this reference. But, the CBM policy was not considered about the unit's RUL prediction.

2.4. Repairable Policy. Many customers began to not only rely on the supplier's resupply. They consider developing the ability in repairing units to decrease the order cost and inventory delay time. To the best of our knowledge, there is little research on the repairable policy. In the formerly discussed reference, they mainly talk about the consumption units. Some of the research studies brought up the repairable policy. Zhou et al. [36] studied the joint optimization of the imperfect CBM and spare provisioning policy for deteriorating systems. The model is considered about the unit's repairment but does not model the repairable ability. In some references [37], the units or maintenance demand obeys any distribution and did not adopt repairable policy into the CBM policy.

When the system finishes repairing, the repaired units will be changed to spare parts. Since then, the structure of the inventory source has changed. Its sources conclude the resupply and repairable policy. The optimized variables concluding the check cycle, preventive threshold, and maximum inventory are changed. So, the corresponding variables should be optimized again. With the resupply parameters changing, the optional decision combination will change. However, there is little research on the effect of resupply parameters in the joint policy. Furthermore, as two sources of spare parts, resupply and repairable policies affect each other. But, the effect is not known.

From the former discussion, we can conclude that there are few studies on the joint optimization of the CBM and inventory policy based on the RUL prediction for a multiunit system. Furthermore, the research on the model of repairable ability in the CBM policy is little. So, we will discuss them in-depth in this article and analyze the characteristic of repairable policy in joint optimization.

\section{Notations and Assumptions}

3.1. Notations. (1) The used notations are given in Table 1.

(2) The notations for decision variables are given in Table 2.

3.2. Assumptions. According to the background and requirements for the policy, the following assumptions are made:

(1) In order to describe all kinds of uncertain failure deterioration degrees (the deterioration degrees in the event of unit failure), the modeling method of failure in this article can make up the gap between random failure and continuous deterioration levels.

(2) This customer has many different styles of equipment, and each of them has the same unit that plays an important role in it. Because the monitoring units are similar, their failure threshold and preventive threshold are the same.

(3) The performance degradation process of this part conforms to the condition of the Wiener process. The performance level of spare parts in stock does not decrease.

\section{Materials and Methods}

4.1. Condition-Based Maintenance with RUL Prediction. In industrial practice, the customer's maintenance department (MD) often replaces failing units directly in the result that the delay will cause high downtime loss. According to the traditional CBM policy, there are two main conditions for replacing units. One is replacing unit after failure $\left(E_{k}^{\tau} \geq L_{f}\right)$. It is called "corrective replacement." The other one is the threshold to replace. When a unit's monitoring value reaches the preset threshold $\left(E_{k}^{\tau} \geq L_{p}\right)$, it will be replaced previously to prevent the unit failed in the check cycle. However, with the development of sensors, the applicant of data-driven technology becomes deeper and deeper. The conditions of replacement develop to the third method, which is RUL prediction. After checking unit $k$ 's monitoring data at current check time $t^{\tau}$, the system will predict its RUL. 
TABLE 1: The used notations.

\begin{tabular}{|c|c|c|}
\hline Symbol & Meaning & Unit \\
\hline k & Index of the unit in its equipment owned by the customer $(k=1,2,3, \ldots, K)$ & \\
\hline$\tau$ & Index of check cycles $(\tau=0,1,2,3, \ldots, \Psi)$ & \\
\hline$n$ & Index of repair table $(n=1,2,3, \ldots, N)$ & \\
\hline$L_{f}$ & Failure threshold & \\
\hline$m$ & Index of historical monitoring time point to build the model of the Wiener process $(m=1,2,3, \ldots, M)$ & \\
\hline$t_{k}^{m}$ & Monitoring time of unit $k$ in period $m$ to build the model of the Wiener process & \\
\hline$E_{k}^{m}$ & Historical performance degradation value of unit $k$ in period $m$ to build the model of the Wiener process & \\
\hline$\mu$ & Mean value of the Wiener process & Use cycle \\
\hline$\sigma$ & Magnetic moment of the Wiener process & \\
\hline$E_{k}^{\tau}$ & Monitoring data of unit $k$ in period $\tau$ & \\
\hline $\mathrm{Tk} \tau$ & Predicted residual life of unit $k$ in period $\tau$ in theory & Use cycle \\
\hline$T$ & Unit's lifetime & \\
\hline$t^{\tau}$ & System check time in period $\tau$ & Use cycle \\
\hline$C_{c}$ & Unit check cost & yuan \\
\hline$C_{p r}$ & Unit cost of preventive maintenance & yuan \\
\hline$C_{c r}$ & Unit cost of corrective maintenance & yuan \\
\hline$C_{f}$ & Unit cost loss per unit per unit time incurred by the unit in the failed state & yuan \\
\hline$C_{i}$ & Unit inventory cost & yuan \\
\hline$C_{o}$ & Unit ordering cost & yuan \\
\hline$C_{r b}$ & Unit repair table building cost & yuan \\
\hline$C_{r}$ & Unit repairing cost & yuan \\
\hline$s$ & Current inventory level & yuan \\
\hline$R^{r}$ & Quantity of spare parts replenishments & \\
\hline$R^{n}$ & Quantity of spare parts repairing in repair table $n$ & \\
\hline$T^{o}$ & Inventory delay time & Use cycle \\
\hline$t^{r}$ & Ending time of resupply in current resupply period & Use cycle \\
\hline$t^{o n}$ & Start working time of repair table $n$ & Use cycle \\
\hline$T^{o n}$ & Interval time of inventory delay in resupply period $\tau_{i}$ & Use cycle \\
\hline$t^{r n}$ & Ending working time of repair table $n$ & Use cycle \\
\hline$N_{c}$ & Total number of checking units & \\
\hline$N_{p r}$ & Total number of preventive replacements & \\
\hline$N_{c r}$ & Total number of corrective replacements & \\
\hline$N_{o}$ & Total number of ordering units & \\
\hline$N_{f}\left(t^{\tau}\right)$ & Number of failed units before corrective replacements are implemented over period $\tau$ & \\
\hline$N_{i}\left(t^{\tau}\right)$ & Number of inventories before replacing over period $\tau$ & \\
\hline$N_{r}$ & Total number of repaired units over period $\tau$ & \\
\hline$E C_{\infty}$ & Average cost per unit per unit time over an infinite time span (i.e., the average cost rate) of the considered system & \\
\hline$E C_{\min }$ & Minimum cost rate & \\
\hline
\end{tabular}

Table 2: The notations for decision variables.

\begin{tabular}{lcc}
\hline Symbol & Meaning & Unit \\
\hline$L_{p}$ & Preventive replacement threshold & \\
$T_{c}$ & Interval time between two adjacent check & Use cycle \\
$S$ & cycles & \\
\hline
\end{tabular}

In reference [5], the mean and variance values of the unit's lifetime $T$ is known by the formula derivation of the Wiener process, as follows:

$$
\begin{aligned}
& E(T)=\frac{L_{f}}{\mu}, \\
& D(T)=\frac{L_{f} \sigma^{2}}{\mu^{3}} .
\end{aligned}
$$

Based on equations (1) and (2), because the density functions of the unit' lifetime and RUL are similar, the system can predict units' RUL $T_{k}{ }^{\tau}$ as follows:

$$
T_{k}^{\tau}=\frac{L_{f}-E_{k}^{\tau}}{\mu} .
$$

However, $\mu$ and $\sigma$ should be known. They can be calculated by maximum likelihood estimation, as follows:

$$
\begin{aligned}
L\left(\mu, \sigma^{2}\right) & =\prod_{k=1}^{K} \prod_{m=1}^{M} \frac{1}{\sqrt{2 \sigma^{2} \pi \Delta t_{k}^{m}}} \exp \left[-\frac{\left(\Delta E_{k}^{m}-\mu \Delta t_{k}^{m}\right)^{2}}{2 \sigma^{2} \pi \Delta t_{k}^{m}}\right], \\
\widehat{\mu} & =\frac{\sum_{k=1}^{K} E_{k}^{m}}{\sum_{k=1}^{K} t_{k}^{m}}
\end{aligned}
$$




$$
\widehat{\sigma}=\frac{1}{K \cdot M}\left[\sum_{k=1}^{K} \sum_{m=1}^{M} \frac{\left(\Delta E_{k}^{m}\right)^{2}}{\Delta t_{k}^{m}}-\frac{\left(\sum_{k=1}^{K} E_{k}^{m}\right)^{2}}{\sum_{k=1}^{K} t_{k}^{m}}\right],
$$

where $\Delta E_{k}{ }^{m}$ is the difference between $E_{k}{ }^{m}$ and $E_{k}{ }^{m-1}, \widehat{\mu}$ is the maximum likelihood estimation in mean value, and $\widehat{\sigma}$ is the maximum likelihood estimation in magnetic moment.

When its RUL is less than the check cycle $\left(T_{k}{ }^{\tau}<T_{c}\right)$, a conclusion can be made that unit $k$ cannot stay working over the last check cycle. Therefore, the system will prepare to replace it at $t^{\tau}$. Comparing with the preventive threshold replacement, the last condition is similar to it. They just replace the corresponding unit. Therefore, it can be called "preventive replacement" together. As for the corrective replacement, an important unit can result in the surrounding units' failure. Therefore, unit corrective replacement cost is higher than preventive replacement $\left(C_{c r}>C_{p r}\right)$. For example, we discussed two units' main checking process, as shown in Figure 1.

In Figure $1, E_{1}{ }^{1}$ that is the current degradation value of the first unit at $t^{1}$, which is the first check time, is lower than $L_{p}\left(E_{1}{ }^{1}<L_{p}\right)$. But, the predicted residual life $T_{1}{ }^{1}$ is less than $T_{c}$ $\left(T_{1}{ }^{1}<T_{c}\right)$. Therefore, the unit will be replaced. Turn to $t^{2}$ which is the ending time of period 2 . The degradation value $E_{1}^{2}$ that is the current degradation value is also lower $\left(E_{1}{ }^{2}<L_{p}\right)$. Different from the former period, the predicted residual life $T_{1}^{2}$ is higher than $T_{c}\left(T_{1}^{2}>T_{c}\right)$. Therefore, it does not need be replaced. However, turning to $t^{3}$, the situation is similar to that at $t^{1}$. The unit is going to be replaced as well.

Next, we obtain the second unit. At $t^{1}, E_{2}{ }^{1}$ that is the current degeneration value of the second unit is lower than $L_{p}\left(E_{2}{ }^{1}<L_{p}\right)$. Its predicted residual life $T_{2}{ }^{1}$ is higher than $T_{c}$ $\left(T_{2}{ }^{1}>T_{c}\right)$. So, it does not need be replaced. However, in the interval time between $t^{1}$ and $t^{2}$, there is a failure in this unit. It caused the breakdown loss. But the unit will not be replaced because it is not at check time. It did not begin the corrective replacement until it is at $t^{3}$. Turning to the next check time, $E_{2}{ }^{3}$ is lower than $L_{p}\left(E_{2}{ }^{3}<L_{p}\right)$. But, $T_{2}{ }^{3}$ that is the current predicted residual life is less than $T_{c}\left(T_{2}{ }^{3}>T_{c}\right)$. The unit will be replaced.

4.2. (S-1, S) Policy. When a unit is under replacement, MD will check whether there is inventory in stock. If yes, the replacement process will go on. According to the characteristic of the $(S-1, S)$ policy, the resupply applicant will be sent out after replacement happened once. On the contrary, if there is no inventory in stock, the unit's replacement will be delayed. The unit's delay will be marked either.

Before checking units, the system will check whether a resupply arrives. When a resupply arrives, MD will take them into stock. At the same time, it will check whether there is a delay unit. If yes, that unit will be replaced immediately. On the other hand, many researchers did not discuss the replacement sequence. In order to avoid downtime loss as much as possible, MD decides to replace failure units firstly after checking units. Take the condition-based maintenance process of two units mentioned in Figure 1 as an example.

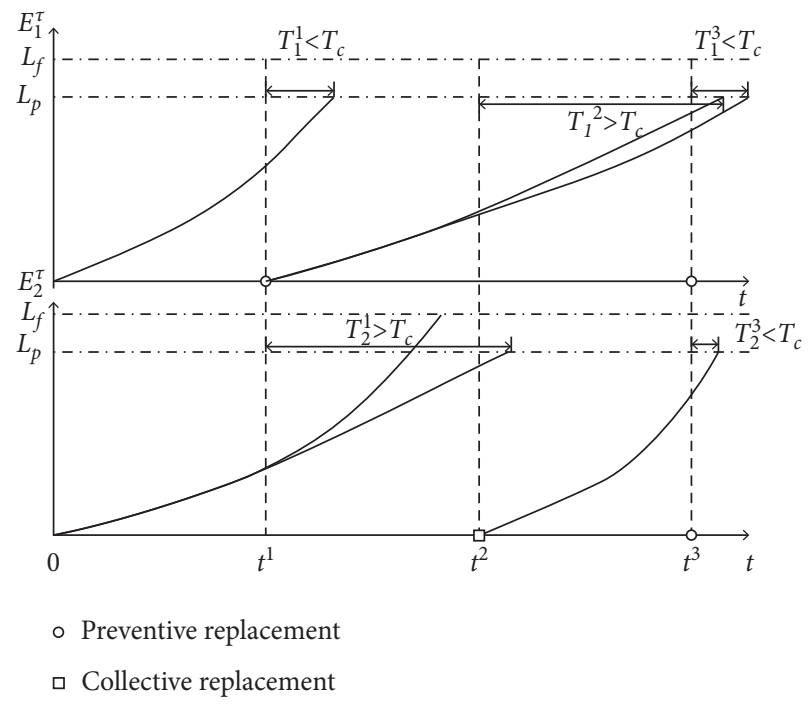

Figure 1: Two units' main checking process.

As shown in Figure 2, the first resupply application has been sent at $t^{1}$. When the application is sent, $t^{1}$ that is the current check time is the resupply start time. Spending $T^{o}$ is the inventory delay time, it gets to $t^{r}$ which is the resupply arriving time. At this time, the first resupply has finished. Turning to $t^{3}$, the second resupply application is sent. The process is the same as the former time. As a result, the arriving time in every period will be obtained.

4.3. Repairable Policy. In the system, the policy is introduced the queuing theory based on the original policy. According to the Kendall notation [38], the joint model adopts " $X / Y / c$ / $\infty / m$ " model for the repairable policy. After a unit is replaced, it can be repaired. However, not all replaced units can be repaired. There are two previous conditions. One is the unit's state. When the unit is failed, it has been damaged destructively so that there is no point in repairing it. Therefore, these units will not be repaired after corrective replacement. On the contrary, units after preventive replacement will be taken into repairing tables to repair. The other is the quantity of repairing tables $(n)$. Before the replaced unit is taken into repairing table, MD will check whether there is a spare working table. If yes, the repairing process will go on. Otherwise, these units will be given up.

After repairing, these units will be changed into spare parts into stock. Turn to the next check time. MD will replace units by using the resupply spare part firstly. This step can increase the unit's working time and increase replacement time as much as possible. Furthermore, the repair table should be built previously. Therefore, MD should spend the building cost previously. The part of the cost that concludes the building cost and repairing cost is rather higher than the ordering cost in the previous simulation time span. However, with it going, the cost of the repairable policy will be lower than the ordering cost. Therefore, the set number of repair tables will affect $E C$ and the optional decision combination. 


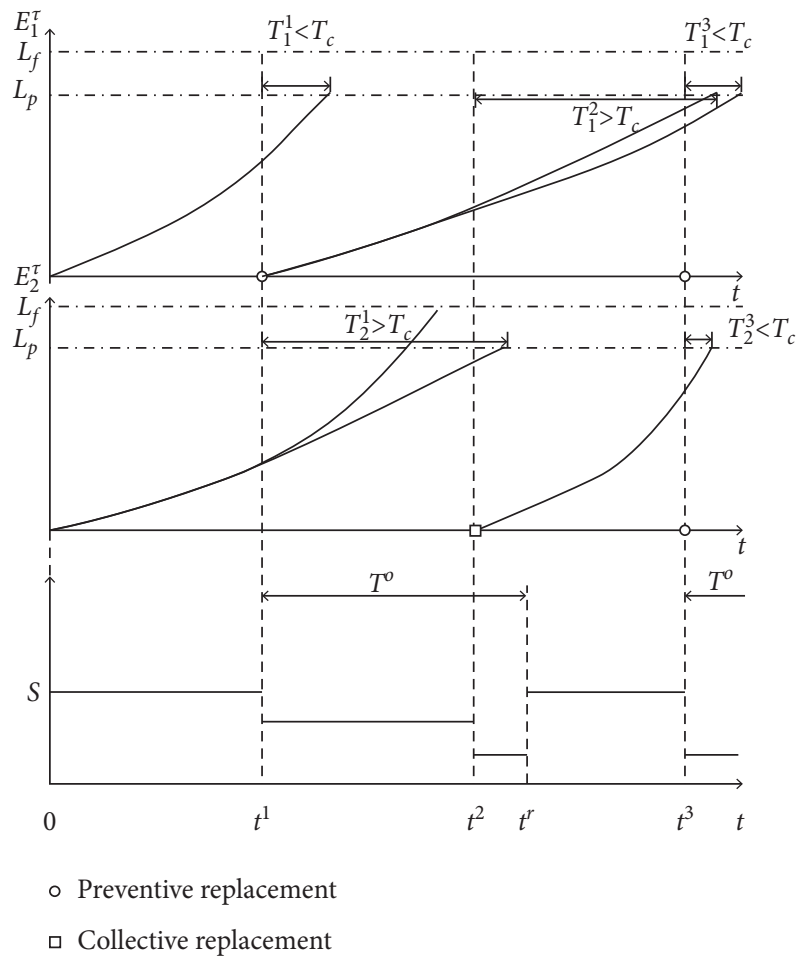

Figure 2: Analysis process in maintenance and inventory for two units.

According to the former discussion, the decision variables will be optimized again after the joint policy adopted the repairable policy. For a slowly deteriorating unit, the too frequent inspection cannot provide a more accurate data basis for the life prediction. On the other hand, it may affect the fitting speed of fault function based on the Wiener process because of the large amount of data. At the same time, this behavior will increase the cost of testing resulting in excessive cost. On the contrary, it may increase failure possibility over the next check cycle that $T_{c}$ is too large so that it could lose the significance of preventive replacement. Secondly, if $L_{p}$ is too high, it will increase the time and the ordering cost. Apart from these aspects, the increasing maximum inventory could affect the inventory cost as well. Furthermore, the decreasing inventory may cause the delayed loss so that the availability of equipment will be affected. In conclusion, the joint policy of maintenance and inventory ordering focuses on adjusting the decision variables that are $T_{c}, S$, and $L_{p}$ to control the costs of preventive replacement, corrective replacement, check and ordering, and even delay loss. It may reduce the total cost as much as possible to obtain the optional decision combination by optimization.

There are mainly two calculating methods. One is establishing an accurate set of formulas after a complete mathematical derivation $[25,38]$. But it is difficult to use explicit expressions to clearly express the relationship between objective functions and decision variables. Then, the joint optimization for the multiple identical parts in this article is a logical process triggered randomly at the discrete time by the change of decision variables. The other is the Monte Carlo simulation. It can solve this kind of problem well. By adjusting the decision variables and analyzing a large number of simulations, the value distribution of the objective function in this interval of time is obtained to get the optional joint policy. The objective function adopted the expense cost rate $\left(E C_{\infty}\right)$ to calculate the joint model. It is argued that the target of $E C_{\infty}$ is minimum as follows:

$$
\begin{aligned}
\min \quad E C_{\infty} & =\min f_{E C}\left(T_{c}, S, L_{p}\right), \\
\text { s.t. } t^{\tau} & =\tau \times T_{c}, \\
\tau & =1,2,3, \ldots, \psi, \\
T_{c} & >0 \\
S & \in N^{+} \\
L_{p} & >0 .
\end{aligned}
$$

Due to the complexity of the joint policy, it is difficult to derive the analytical formulation of the function $f_{E C}\left(T_{C}, S\right.$, $\left.L_{p}\right)$. However, the average cost per unit time over an infinite time span (i.e., the average cost rate) of the considered system can also be simply represented as follows:

$$
E C_{\infty}=\lim _{T \longrightarrow \infty} \frac{C_{c} \cdot N_{c}+C_{p r} \cdot N_{p r}+C_{c r} \cdot N_{c r}+C_{o} \cdot N_{o}+C_{f} \cdot \sum_{\tau=1}^{\psi} N_{f}\left(t^{\tau}\right)+C_{i} \cdot \sum_{\tau=1}^{\psi} N_{i}\left(t^{\tau}\right)+C_{r} \cdot N_{r}+C_{r b} \cdot N}{T \cdot K} .
$$

The way to evaluate the average cost rate $E C_{\infty}$ based on equation (9) by simulation will be described in the following discussion. In this study, the time value of money is not considered.

4.4. Building the Simulation Model. According to the proposed joint policy, the Monte Carlo simulation method is used to evaluate $E C_{\infty}$. By simulating a large number of system life histories, it can estimate the ensemble averages of the concerned quantities for the calculation. Each simulated history can be considered as a virtual experiment in which the unit deterioration processes are followed in their evolution over the check cycle. The Monte Carlo simulation can be divided into the simulation with variable time increments and the simulation with fixed time increments. The latter will be used in this paper.

In order to illustrate the model clearly, the used marks are listed in Table 3. 
TABLE 3: The used marks in the simulation model.

\begin{tabular}{lc}
\hline Symbol & Meaning \\
\hline$T$ & Code to signify whether the unit $k$ is failed at the current discrete time before any decision is made \\
$S F_{k}$ & Code to signify whether a preventive replacement of unit $k$ is implemented at the current check time \\
$S P P R_{k}$ & Code to signify whether a corrective replacement of unit $k$ is implemented at the current check time \\
$S P C R_{k}$ & Code to signify whether an order is outstanding or not \\
$O R$ & Number of spare parts that finish repairing over the current check cycle \\
$R^{r a}$ & Number of spare parts entering into stock before checking units' states \\
$R^{\text {int }}$ & Stock level (the number of spare units in inventory) at the current instant before any decision is made \\
$s^{-}$ &
\end{tabular}

4.4.1. Modeling Deterioration Based on the Wiener Process and Replacement of Units. At first, according to the Wiener process, the process requires to model the deterioration of units, as follows:

$$
E_{k}^{\tau}=E_{k}^{\tau-1}+\Delta E_{k}^{\tau}
$$

where $\Delta E_{k}^{\tau}\left[\Delta E_{k}^{\tau} \sim\left(T_{c} \mu, T_{c} \sigma\right)\right]$ that obeys normal distribution is the random increment between $E_{k}^{\tau}$ and $\Delta E_{k}^{\tau-1}$ as follows

$$
\Delta E_{k}^{\tau}\left(T_{c}\right)=\mu \cdot T_{c}+\sigma \cdot B\left(T_{c}\right) .
$$

According to the unit's monitoring value, the unit's state can be known. Besides, the unit's RUL $T_{k}{ }^{\tau}$ is also known according to equations (1) and (2). Resulting from preventive replacement cost is lower than corrective replacement cost $\left(C_{p r}<C_{c r}\right)$, the number of spare parts consumptions of a preventive and corrective replacement should be marked separately. In order to mark the unit's state, we set "SPPR ${ }_{k}$ " and "SPCR ${ }_{k}$." The amount of preventive and corrective replacements will be marked. The replacement process is as follows:

$$
\begin{aligned}
S F_{k} & = \begin{cases}1, & E_{k}^{\tau} \geq L_{f}, \\
0, & E_{k}^{\tau}<L_{f},\end{cases} \\
\text { SPCR }_{k} & = \begin{cases}1, & \left(S F_{k}=1\right) \cup(s>0), \\
0, & \text { others, }\end{cases} \\
S F_{k} & = \begin{cases}0, & S P C R_{k}=1, \\
S F_{k}, & \text { others, }\end{cases} \\
S P P R_{k} & = \begin{cases}1, & \left(S F_{k}=0\right) \cup\left\{\left(E_{k}^{\tau}>L_{p}\right) \cap\left[\left(E_{k}^{\tau} \leq L_{p}\right) \cup\left(T_{k}^{\tau}<T_{c}\right)\right]\right\} \cup(s>0), \\
0, & \text { others. }\end{cases}
\end{aligned}
$$

(a) When the deterioration value reaches $L_{f}$, the unit is a failure, as shown in equation (12). If there is inventory, it will be correctively replaced, as shown in equation (13). Except that, unit $k$ 's failure state will be updated to " 0 " after it is replaced. On the contrary, the state will be retained, as shown in equation (14).

(b) When deterioration value is up to $L_{p}$, the unit needs a preventive replacement. If there is inventory, it will finish replacement. The process is shown in equation (15).

(c) When the deterioration value is lower than $L_{p}$, the unit will be checked deeply. The system will predict its RUL. If $T_{k}{ }^{\tau}$ is shorter than $T_{c}\left(T_{k}{ }^{\tau}<T_{c}\right)$; the unit needs a preventive replacement either. If there is inventory, it will finish replacement. The process is shown in equation (15).

(d) When there is little inventory, the units that need a corrective replacement will be delayed. However, the units that need a preventive replacement will continue to be used. Its deterioration value will remain the same, as shown in equation (14).

When the unit is replaced, its deterioration value will be updated. The unit's deterioration value would be recalculated again, and the delay unit will remain in the current state. The other working units' deterioration value will change as shown in equations (10) and (11). The main process is as follows:

$$
\Delta E_{k}^{\tau+1}= \begin{cases}E_{k}^{\tau}, & \left(S F_{k}=1\right) \cup\left(S P C R_{k}=0\right), \\ -\Delta E_{k}^{\tau}+\mu T_{c}+\sigma, & \left(S P P R_{k}=1\right) \cap\left(S P C R_{k}=1\right), \\ \mu T_{c}+\sigma, & \text { others. }\end{cases}
$$

After replacement, the current inventory will also be updated. The process is as follows: 


$$
s= \begin{cases}s-1, & \left(S P P R_{k}=1\right) \cap\left(S P C R_{k}=1\right), \\ s, & \text { others. }\end{cases}
$$

4.4.2. Modeling Spare Order. There are two sources for replenishing spare parts. The main one is resupply. Once the inventory decreases, the resupply applicant would be sent at once. But the delay time will be over some check cycles. In order to mark the resupply applicant, $O R$ is set. The main updated process is as follows:

$$
O R= \begin{cases}1, & (s<S) \cap(O R=0) \\ 0, & \text { others }\end{cases}
$$

MD will not only focus on the inventory but also check whether a resupply applicant outstands in the system. If there is a resupply applicant outstanding, the next one will not be sent out. From then on, the arriving time will be marked. $t^{r}$ is determined by the applicant sending time $t^{\tau}$ and inventory delay time $T^{\circ}$, as follows:

$$
t^{r}=T^{o}+t^{\tau}
$$

The number of resupply spare parts $R^{r}$ is determined by $\Delta s$, as follows:

$$
R^{r}=\Delta s=S-s,
$$

where $\Delta s$ is the difference between the current inventory and maximum inventory. Resulting from it, the system will check whether a resupply arrived before checking units. The checking process is as follows:

$$
R^{\text {int }}= \begin{cases}R^{r}, & t^{r} \bmod T_{c} \leq \tau, \\ 0, & \text { others. }\end{cases}
$$

4.4.3. Modeling Repair Queues. Another source is the repair table. According to the discussion of repairable policy, MD will be ready to take units that are replaced preventively into repairing tables. However, it will check whether there is a spare repair table in the system. Generally, the repair capacity is relatively limited. Repairs can only be carried out for a certain batch of units. The main checking process is as follows:

$$
n= \begin{cases}n, & t^{r n}=0, \\ n+1, & t^{r n} \neq 0 .\end{cases}
$$

The system will check the whole repair tables. While there is an available repair table, units will be taken into this repairing table. The repairing number in this time is the amount of the units, which are replaced preventively, as follows:

$$
R^{n}=\sum_{k=1}^{K} S P P R_{k}
$$

Besides, when a repairing table accepts units, the start time $t^{o n j}$ of this repairment process will be marked. With it going $T^{\text {onj }}$, the ending time $T^{r n j}$ will be known, as follows:

$$
t^{r n}=t^{o n}+T^{o n}
$$

After repairment, the replaced units will be changed to spare parts. Along with checking resupply applicants, The system will check whether any repair table finishesrepairing. If yes, the repaired units will be taken into stock. The main decision process is as follows:

$$
R^{r a}= \begin{cases}R^{r a}+R^{n}, & t^{r n} \bmod T_{c} \leq \tau, \\ R^{r a}, & \text { others. }\end{cases}
$$

The ending time of the repair table that finishes repairing will be initiated, as follows.

$$
t^{r n}= \begin{cases}0, & t^{r n} \bmod T_{c} \leq \tau, \\ t^{r n}, & \text { others. }\end{cases}
$$

After checking resupply and repair tables, MD will take the spare parts into stock. The inventory will increase as follows:

$$
\begin{aligned}
R^{\text {int }} & =R^{\text {int }}+R^{r a}, \\
s & =s+R^{\text {int }} .
\end{aligned}
$$

4.4.4. Evaluating the Average Cost Rate. After all replacements are carried out at the current check time, $N_{c}, N_{p r}, N_{c r}$, $N_{o}$, and $N_{r}$ must be accumulated, and $N_{f}\left(t^{\tau}\right)$ and $N_{i}\left(t^{\tau}\right)$ should be recorded, represented as follows:

$$
\begin{aligned}
N_{c} & =N_{c}+K, \\
N_{p r} & =N_{p r}+\sum_{k=1}^{K} S P P R_{k}, \\
N_{c r} & =N_{c r}+\sum_{k=1}^{K} S P C R_{k}, \\
N_{o} & =N_{o}+R^{r}, \\
N_{r} & =N_{r}+R^{r a}, \\
N_{f}\left(t^{\tau}\right) & =\sum_{k=1}^{K} S F_{k}, \\
N_{i}\left(t^{\tau}\right) & =s^{-} .
\end{aligned}
$$

After finishing the simulation of all events over the simulation time span $T$, the average cost rate can be derived based on equation (8).

For further description of the Monte Carlo simulation, the main flow diagram of joint policy is as shown in Figure 3. The first step "initialize policy parameters" is to input the system parameters $C_{c}, C_{p r}, C_{c r}, C_{f}, C_{i}, C_{o}, C_{r}, K, \Psi, \Phi, N, T, M$, $L_{f}, T^{o}$, and set $\tau=0, E_{k}{ }^{\tau}=0$ (where $k=1,2,3, \ldots, K ; \tau=0,1,2$, $3, \ldots, \Psi) ; t^{r}=0, t^{o n}=0$ (where $\left.n=1,2,3, \ldots, N\right)$; and $s=S$, $S F_{k}=0, S P P R_{k}=0, S P C R_{k}=0, O R=0, R^{r a}=0, R^{\text {int }}=0$, and $s^{-}=0$, where $k=1,2,3, \ldots, K$. The simulation time span $T$ should be set large enough.

In the former discussion, the effect on the cost rate has been addressed with the decision variables changing. With the application of the Monte Carlo method above, the cost rate can be evaluated given a specific set of the decision 

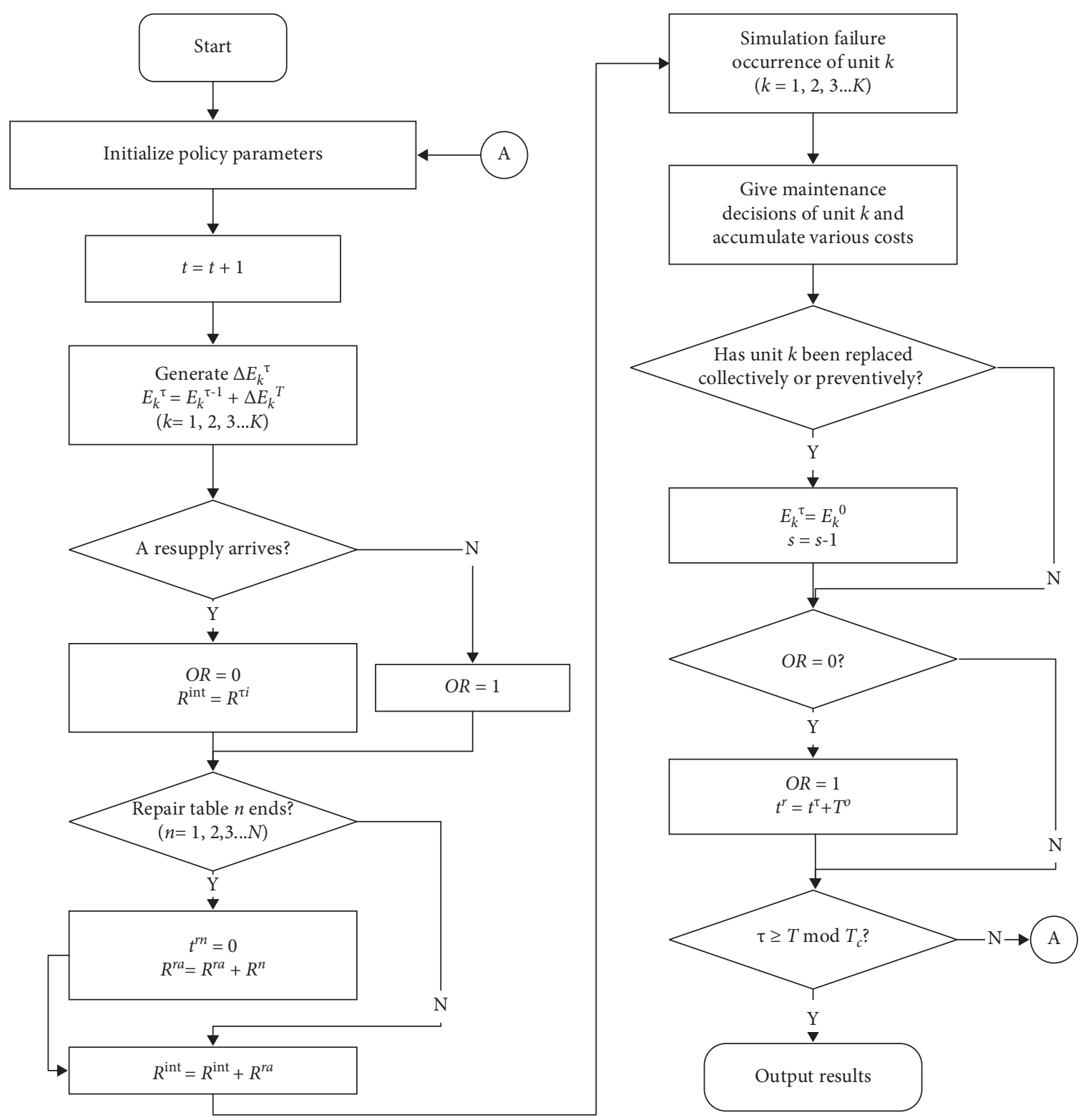

FIGURE 3: Flow diagram for the simulation program.

variables $\left(L_{p}, T_{c}\right.$, and $\left.S\right)$ under the proposed joint policy. The aim is to minimize the cost rate and obtain the optional values of the decision variables combination $\left(L_{p}^{*}, T_{c}^{*}\right.$, and $S^{*}$ ). Therefore, a particle swarm optimization (PSO) algorithm is used to determine the optional decision combination.

4.5. PSO Algorithm. Metaheuristic algorithms include particle swarm optimization algorithm, genetic algorithm, simulated annealing algorithm, artificial bee swarm algorithm, and so on [39]. In this article, the units' states are iterated as the period changes in one simulation. When the simulation iterates many times, the model parameters are iterated many times either, which makes the optimization of the algorithm more difficult. Furthermore, compared with analytical calculation, the conditions and calculation processes of the simulation problem are more complex. The PSO algorithm can solve this problem well [40]. The PSO algorithm is one of the most widely used algorithms in multiperiod model optimization [41]. It is paid a high attention by researchers because of its easy implementation, high precision, and fast convergence. It has been demonstrated by its superior algorithm characteristics in practical applications. The PSO algorithm is used to optimize the joint model to obtain the optional decision combination $\left(T_{c}^{*}, S^{*}\right.$, and $L_{p}^{*}$ ).

At first, the system will generate many groups of particle swarm. There are three particles in a group standing the decision parameters $\left(T_{c}, S\right.$, and $\left.L_{p}\right)$ separately. All groups enter the simulation process according to Figure 3 . The simulation results of all groups will be marked. The global optional result is identical, and the global optional group is marked. The system will find the optional group whose result 
is minimum through the PSO algorithm. This is an iteration of optimization. The next optimization will begin. New groups of particles will be generated. The above process should be repeated. The system will compare with the new optional result and the former. When the new one is less than the former, the global optional result is the same as the new one, and the global optional group is marked. When the new one is higher than the former, the global optional result does not change. The optimization will not repeat until the iteration reaches the maximum iteration. The main process is as shown in Figure 4 (Algorithm 1).

\section{Case Study}

5.1. Case Description. In automotive engines, because of the core position of the engine in the composition of the vehicle, especially racing cars, the fault monitoring of the engine (internal combustion engine) and the subsequent maintenance procedure have always been the important contents that researchers pay close attention to. From the view of the maintenance department, the internal combustion engine has two main characteristics. First is its mechanical structure being complex, in order to ensure the tightness of the cylinder and the completeness of combustion. For example, the cylinder of a gasoline internal combustion engine is generally made by pouring with an integrated mold. Apart from it, there are a lot of parts loading in the engine, such as bearings, pistons, and flywheels. The subtly hidden trouble caused by any one part may lead to the failure of the engine. The other is that most of the main body of the automobile engine is generally embedded in the automobile interior so that the general self-test program cannot check whether there is an obvious fault on the outside of the engine. Furthermore, it is too hard to check the interior of the engine. Therefore, the German Volkswagen Group, General Motors of the United States, Toyota of Japan, and other companies gradually began to use electronic monitoring and control technology to carry out real-time monitoring of the engines and other key components. On the one hand, the central control system notifies the maintenance team belonging to the fleet. On the other hand, it may send the fault analysis report to the customer center belonging to the depot through the remote platform as well. When the vehicle enters the maintenance workshop, the maintenance personnel can download the monitoring data from the central control system for data analysis, so as to provide options for the later maintenance and provide suggestions for the improvement of the car to the $\mathrm{R} \& \mathrm{D}$ department of the car company.

According to the data from a fleet, which is in the interval of time from 2012 to 2017, the team is mainly responsible for the maintenance and modification of 10 vehicles in the fleet, including training, racing, and spare vehicles. All vehicles are monitored for the part of the engine. According to reference [42], the data was preprocessed. Then, the data is processed based on performance degradation modeling. The process does not belong to the main content of this article, so it will not be elaborated here. The result is as Table 4 .
In Table 4, columns 2 and 3 are the third- and fourthorder center distances, respectively, in order to check the value of $k$-th skewness and kurtosis, and columns 4 and 5 are the parameter values of the statistical test.

$\alpha$ that is the significance level for determining whether the deterioration process that can be express by the Wiener process is equal to 0.05 . If the value of $\left|\mu_{1}\right|$ and $\left|\mu_{2}\right|$ are both less than $Z_{\alpha / 4}$, the data can be used by this method. It is known that $Z_{\alpha / 4}$ is equal to 2.25. Therefore, the values of columns 3 and 4 are not higher than 2.25. After checking, the degradation process can be expressed by the Wiener process.

Maintenance and inventory costs have fluctuated over the past six years. Resulting from it, we considered the impact of economic purchasing power fluctuations on modeling in this article to process the cost data of six years. The calculated costs of every aspect are given in Table 5 .

Considering the setting of model parameters, $T$ that is the whole optimization time is equal to 20,000 use cycle. $C_{c}$ that is the unit check cost is equal to 500 yuan. When the unit's monitoring value is less than $L_{f}$, it is a failure. After it was finished a corrective replacement, the system would cost 30,000 yuan that is the unit cost of corrective replacement $C_{c r}$. Its performance would turn back to the initial state. However, there would be a delay loss $C_{f}$ if there is no inventory. It may lead to the cost of personnel, equipment, and reputation because of the inability to carry out daily training, unnormal competitions, and other planned schedule work. It is roughly 50,000 yuan through calculating. In the meantime, the team will replace preventively units whose monitoring value is less than $L_{p}$. It would cost 5,000 yuan that is the unit cost of preventive replacement. The maintenance team is equipped with two repair teams on a daily basis. The team will finish once repairing task in 2,500 use cycle, and $C_{r}$ is roughly equal to 3,000 yuan. MD will build the corresponding repair tables. The unit building cost $C_{r b}$ is 10,000 yuan. The $(S-1, S)$ policy is adopted to manage the inventory. The inventory cost in every check cycle includes the venue fee, the management salary, and the arrangement cost of the inventory environment. It is roughly 3,000 yuan that is the unit inventory cost. It would not send the resupply applicant until there is no consumption. The inventory delay time is about 3,000 use cycle. The ordering cost of unit spare part $C_{o}$ is 4,000 yuan.

5.2. Modeling in Degradation. According to the former discussion, the maximum parameter estimation method is used to calculate the above data. What sets the initial value of state is equal to $75\left(E_{k}^{0}=75\right)$. They are calculated that $\widehat{\mu}$ is equal to -0.01475 and $\bar{\sigma}$ is equal to 0.39985 . The deterioration process of the internal combustion engine can use equation (10) to follow, as follows:

$$
E_{k}^{\tau}(t)=75-0.01475 t+0.39985 B(t) \text {. }
$$

Set " $L=0$." The whole process is simulated by the Monte Carlo method. It is obvious that the monitoring value changes over time, as shown in Figure 5. Furthermore, over time, the offset increases gradually. Except that, it is possible that the predicted value of the next period is higher than the 



Figure 4: Flow diagram for the PSO algorithm.

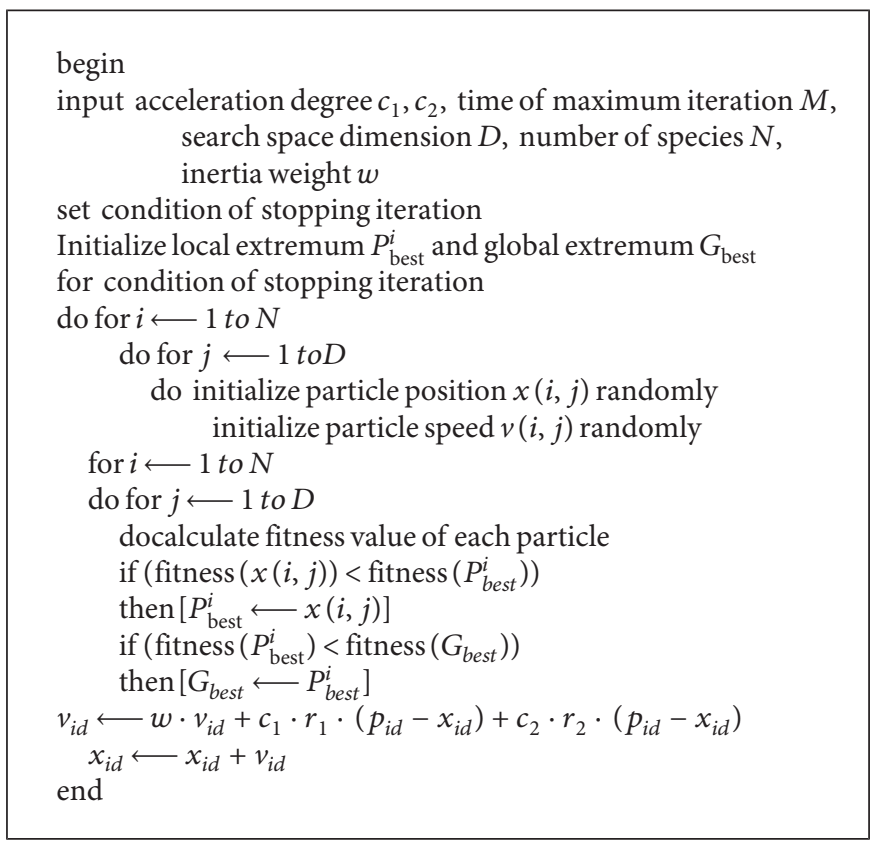

Algorithm 1: Traditional PSO algorithm.

former. The deviation is between the linear functions which is getting larger and larger results in the wider and wider scope of Brownian motion.

According to equation (3), the system would predict its RUL, as shown in Figure 6.

5.3. Result Analysis. By Monte Carlo simulation and the PSO algorithm, the system got the optimal decision combination
[2010, 5, 24]. It is shown that the system should check units in 2010 use cycle. When the unit's data that is monitored by the system in check time is reaching 24 , it will be replaced preventively. The maximum inventory is 5 . Once the inventory decreases, the resupply applicant will be sent. According to the simulation result, the system will finish about 50 preventive replacements and 12 corrective replacements over the simulation time. $E C_{\min }$ is 5.4701 yuan. The convergence process is shown in Figure 7. 
TABLE 4: Statistical results of data analysis.

\begin{tabular}{lcccc}
\hline No. & $g_{1}$ & $g_{2}$ & $\mu_{1}$ & $\mu_{2}$ \\
\hline 1 & 0.4465 & 2.929 & 1.2532 & 0.722 \\
2 & -0.3472 & 2.7418 & 1.0619 & 1.1577 \\
3 & 0.7363 & 3.0332 & 2.0687 & 1.7633 \\
4 & 0.2786 & 2.9975 & 0.7838 & 1.1075 \\
5 & -2.445 & 2.6326 & 0.6725 & 2.0883 \\
6 & 0.0526 & 2.9214 & 0.1487 & 0.6422 \\
7 & -0.082 & 2.7745 & 0.2304 & 0.8263 \\
8 & -0.2252 & 2.7765 & 0.605 & 0.6049 \\
9 & 0.1047 & 2.6556 & 0.2942 & 2.0192 \\
10 & -0.154 & 2.6817 & 0.4327 & 1.7516 \\
\hline
\end{tabular}

TABLE 5: List of costs after processing.

\begin{tabular}{|c|c|c|c|c|c|c|c|c|}
\hline & $C_{c}$ & $C_{p r}$ & $C_{c r}$ & $C_{o}$ & $C_{f}$ & $C_{i}$ & $C_{r b}$ & $C_{r}$ \\
\hline Fitness (Unit: yuan) & 500 & 5,000 & 30,000 & 4,000 & 50,000 & 3,000 & 10,000 & 3,000 \\
\hline
\end{tabular}



Figure 5: Performance degradation curve obtained through simulation.

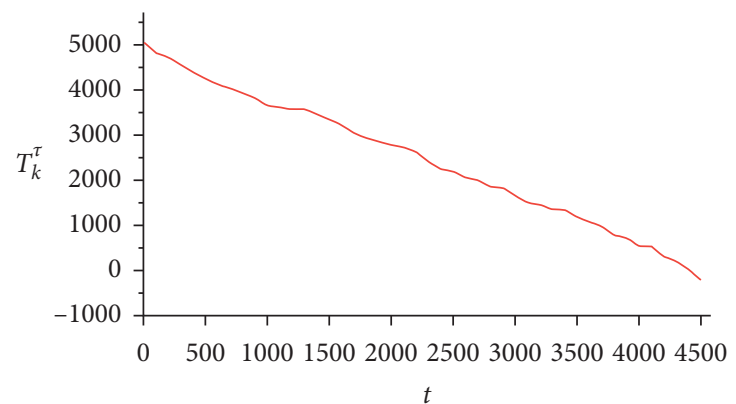

FigURE 6: RUL prediction through simulation.

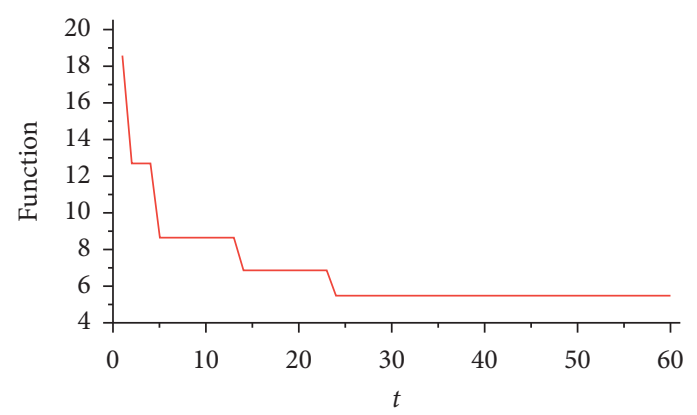

FIgURE 7: Objective function changing process by the PSO algorithm. 
TABLE 6: Sensitivity analysis in the results of joint optimization of the $(s, S)$ policy under conditions, which are the same as the $(S-1, S)$ policy.

\begin{tabular}{lccccc}
\hline No. & $T_{c}$ & $L_{p}$ & $S$ & $s$ & \\
\hline $1^{*}$ & 2010 & 24 & 5 & 4 & $5 C_{\infty}$ \\
2 & 2000 & 24 & 5 & 4 & 6.0076 \\
3 & 2020 & 24 & 5 & 4 & 5.6880 \\
4 & 2010 & 25 & 5 & 4 & 5.6889 \\
5 & 2010 & 23 & 5 & 4 & 5.6806 \\
6 & 2010 & 24 & 6 & 3 & 5.7635 \\
7 & 2010 & 24 & 4 & 5.9045 \\
8 & 2010 & 24 & 5 & 5.6487 \\
\hline
\end{tabular}

*The optional optimization decision combination.

From Figure 7, we can infer that the function curve tends to be smooth in the last convergence curve, and the fluctuation range of the end decreases gradually. So, [2010, 5, 24] is the optimal decision combination.

Except that, according to the former discussion, the ( $S-1$, $S)$ policy is a kind of the $(s, S)$ policy. For showing the advantages of this policy in the joint optimization, we analyzed the sensitivity of the $(s, S)$ policy under the same conditions.

From Table 6, the joint decision combination based on the $(S-1, S)$ policy is the same as that based on the $(s, S)$ policy. The No.1 decision variables combination is the optional one whose $E C_{\infty}$ is the lowest. It is also proved that $[2010,5,24]$ is the optimal decision combination. Furthermore, these optimization results in Table 4 are easy to analyze. When the number of units in the system is up to a certain value, the degree of failure unitsrelatively increases in one check. But, due to the fact that most working units using condition monitoring have a long life and are relatively stable, the probability of failure is low in a certain time range. However, if there is a failure, it is very likely that there will be a lot of units that need to be replaced at the same time. As a result, there is more than one spare part consumed at the same time. Therefore, in this case, it is necessary to resupply spare parts. From the above data and analysis, it is obvious that the joint policy based on the $(S-1, S)$ policy has a great advantage.

Except that, it is known that the efficiency of multiunit optimization is higher than the single-unit optimization. But with the applicant of repairable policy, the conclusion can be inferred again. In Table 7, the comparison between singleand multiunit optimization is given under the same conditions.

From Table 5 and Figure 8, compared with the result of the multiunit optimization, $L_{p}$ is the same. But Tc increases, and $S$ decreases. Limited to the discussion of this case, when $K$ is up to $10, S$ should be set to 10 according to the result of single-unit optimization. As for the multiunit optimization, $S$ is only 5 . While the inventory space is less, $E C_{\infty}$ is up to 6.9869 yuan according to the result of single-unit optimization. However, $E C_{\infty}$ in the multiunit optimization is only 5.6471 yuan. For researching how $K$ will affect the optimization, the optimization results in different values of $K$ are compared. The result is shown in Table 8.

From Table 8, with the number of unit increases, multiunit optimization can save inventory space. While $K$ is increasing, the increasing speed of $S$ is not the same as that of
$K$. For example, when $K=2, S=1$, which is the same as the state when $K=1$. The multiunit optimization makes the separate inventory change into one stock. All of the equipment can use the spare parts in the stock to replace their units. It will decrease the inventory cost and order cost. In the end, $E C_{\infty}$ will decrease.

In references $[43,44]$, the researchers analyzed the influence of parameters on optimization results, such as every kind of cost, replacement threshold, and maximum inventory. There has been a comprehensive sensitivity analysis as far. However, the queuing theory is adopted into the joint policy in this article. One of the most important pieces of research in this article is the repairable policy.

From Table 9, the preventive replacement threshold in the optimization result with repairable policy is lower than that without repairable policy. The other variables are the same. However, $E C_{\infty}$ of the optimization with repairable policy is lower than the optimization without repairable policy. From Figure 9, though with repairable policy, the system should pay the building repair tables cost previously. But $C_{r}$ is lower than $C_{o}$. The number of check time is 9 , and the number of finishing repairing times is 7. A large part of spare parts that rely on the resupply can be gotten by the repair tables. It is about seven ninths. Therefore, $E C_{\infty}$ of the optimization with repairable policy is lower than the optimization without repairable policy. The repairable policy will cost some money previously. However, over multiple periods, the system will save a large part of the cost in ordering by increasing the repairing cost. So, customers can develop its repair teams for its further development.

However, it is not good that more repair tables building. It is a limitation in the number of repair teams. According to our research, the influence on $E C_{\infty}$ through changing the number of repair tables is shown in Table 10.

From Table 10, it is obvious that $E C_{\infty}$ is not decreasing with $N$ decreasing. When $N=2, E C_{\infty}$ is the lowest among the results in Table 10. From Figure 8, the repair policy affects the ordering cost, repair tables building cost, and repairing cost. Therefore, the comparison results among the different repair tables are obtained, as shown in Figure 9.

From Figure 10, when $N=1$, the ordering cost is largely higher than the cost when $N=2$ or 3 . When $N=1$, the number of repair tables working times is 5 , and the number of repaired units arriving times is 4 . The number of spare parts from repair tables increases about two ninths from the state when $N=1$ to $N=2$. This part of spare parts will change from resupply to rely on the repair tables. $C_{r}$ is lower than $C_{o}$. 
TABLE 7: Comparison of optimization results between single- and multiunit systems under the same conditions.

\begin{tabular}{llllr}
\hline Policy & $T_{c}$ & $L_{p}$ & $S$ & \\
\hline Single unit & 2020 & 24 & 1 & 6.9869 \\
Multiple units & 2010 & 24 & 5 & 5.6471 \\
\hline
\end{tabular}

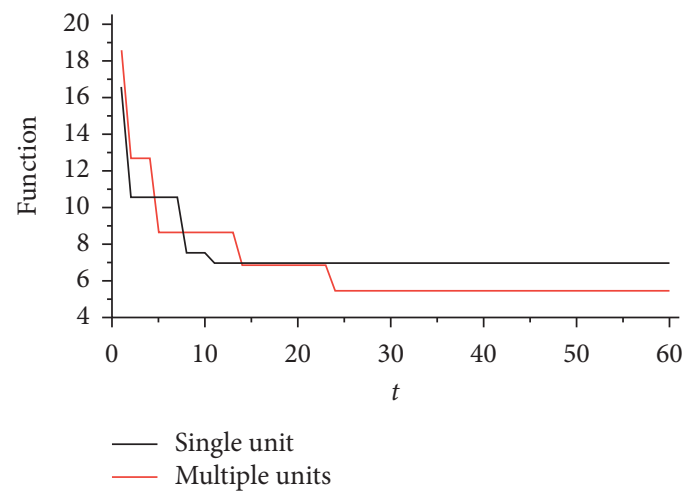

FIGURE 8: Objective function changing process by the PSO algorithm single- and multiunit optimization.

Table 8: Optimization results of different numbers of units in the system under the same conditions.

\begin{tabular}{lclll}
\hline$K$ & $T_{c}$ & $L_{p}$ & $S$ & $E C_{\infty}$ \\
\hline 1 & 2020 & 24 & 1 & 6.9869 \\
2 & 2010 & 25 & 1 & 6.4721 \\
3 & 2020 & 24 & 2 & 6.1633 \\
4 & 2010 & 24 & 2 & 6.0100 \\
\hline
\end{tabular}

TABLE 9: Comparison with the optimization results in the two policies, which are with repairable policy and without repairable policy.

\begin{tabular}{llllr}
\hline Policy & $T_{c}$ & $L_{p}$ & $S$ & \\
\hline Repairable & 2010 & 24 & 5 & 5.4701 \\
No repairable & 2010 & 27 & 5 & 5.7425 \\
\hline
\end{tabular}

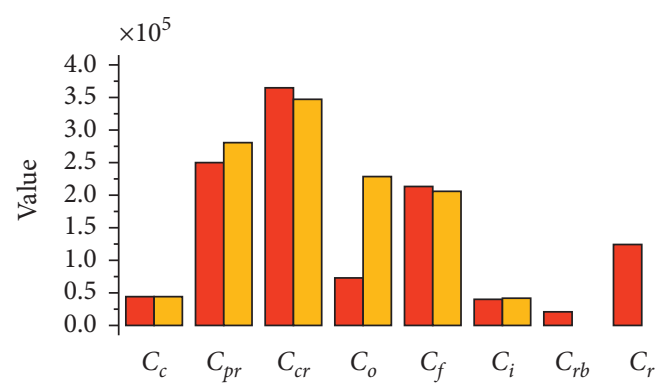

FIGURE 9: Comparison between different kinds of cost with repairable policy and without repairable policy over simulation time span after optimization by the PSO algorithm.

Therefore, $E C_{\infty}$ is decreasing. However, when $N=2$, the number of repair tables working times and the number of repaired units arriving times are the same as the state when $N=3$. The costs between $N=2$ and $N=3$, which does not conclude the repair tables building cost, are the same. But the repair tables building cost is certain. Therefore, $E C_{\infty}$ will be increasing. Above all, increasing $K$ blindly not only has little influence on the optimization result but also causes a waste of resources. On the contrary, reducing $K$ blindly will increase the number of repairable units entering the repair table. Because of the difference between the ordering cost and repairing cost, it can also cause a certain increase in cost. 
TABLE 10: Influence on $E C_{\infty}$ through changing the number of repair tables.

\begin{tabular}{lc}
\hline$N$ & $E C_{\infty}$ \\
\hline 1 & 5.6903 \\
2 & 5.6471 \\
3 & 5.6814 \\
\hline
\end{tabular}



FIgURE 10: Comparison of three kinds of costs (ordering cost, repair tables building cost, and repairing cost) among the different repair tables.

In the end, the repairable policy can reduce $E C_{\infty}$ to a certain extent, but the number of repair resources needs to be arranged reasonably according to the actual situation.

\section{Conclusions}

The center of this article focuses on the joint policy of maintenance and inventory ordering for the multiunit system. Performance degradation model is obtained using widespread life prediction methods based on the Wiener process. Considering the characteristics of small inventory and the small proportion of maintenance budget in the actual industry, the $(S-1, S)$ policy with higher guarantee efficiency and better economic benefit is selected. Adopting the repairable policy, the joint model was built. The joint optimization balances the decision variables that are the check cycle, the maximum inventory, and the preventive replacement threshold to obtain the optional decision combination.

Secondly, the effects of multiunit optimization, the $(S-1$, $S$ ) policy, and repairable policy on the optimization results are investigated to show the advantages of this joint policy. The above research results not only provide optimization solutions for industrial practice but also provide a feasible reference for the follow-up intelligent decision-making in spare parts ordering for the multiunit system in complex maintenance environments.

In this paper, the resupply model can be continued to study under the unstable inventory delay time. In addition, the queuing models in repairable policy can go on studying further.

\section{Data Availability}

The data used to support the findings of this study are available from the corresponding author upon request.

\section{Conflicts of Interest}

The authors declare that there are no conflicts of interest.

\section{References}

[1] G. T. Friedlob and F. J. Plewa, Understanding return on Investment, Wiley, New York, NY, USA, 1996.

[2] Z. Liu and L. Zhang, "A review of failure modes, condition monitoring and fault diagnosis methods for large-scale wind turbine bearings," Measurement, vol. 149, Article ID 107002, 2020.

[3] K. Le Son, M. Fouladirad, A. Barros, E. Levrat, and B. Iung, "Remaining useful life estimation based on stochastic deterioration models: a comparative study," Reliability Engineering \& System Safety, vol. 112, pp. 165-175, 2013.

[4] R. B. Chinnam, "On-line reliability estimation for individual components using statistical degradation signal models," Quality and Reliability Engineering International, vol. 18, no. 1, pp. 53-73, 2002.

[5] G. A. Whitmore and F. Schenkelberg, "Modelling accelerated degradation data using wiener diffusion with A time scale transformation," Lifetime Data Analysis, vol. 3, no. 1, pp. 27-45, 1997.

[6] M. E. Robinson, "Bayesian methods for a growth-curve degradation model with repeated measures," Lifetime Data Analysis, vol. 6, no. 4, pp. 357-374, 2000.

[7] C. Guo, W. Wang, B. Guo, and X. Si, "A maintenance optimization model for mission-oriented systems based on Wiener degradation," Reliability Engineering \& System Safety, vol. 111, pp. 183-194, 2013.

[8] W. Wang, "A stochastic model for joint spare parts inventory and planned maintenance optimisation," European Journal of Operational Research, vol. 216, no. 1, pp. 127-139, 2012.

[9] G. J. Feeney and C. C. Sherbrooke, "The (S-1, S) inventory policy under compound Poisson demand," Management Science, vol. 12, no. 5, pp. 391-411, 1966.

[10] S. Bashyam and M. C. Fu, "Optimization of (s, S) inventory systems with random lead times and a service level constraint," Management Science, vol. 44, pp. S243-S256, 1998.

[11] E. M. Alvarez, M. C. van der Heijden, and W. H. M. Zijm, "Service differentiation in spare parts supply through dedicated stocks," Annals of Operations Research, vol. 231, no. 1, pp. 283-303, 2015.

[12] Z. Zhang, X. Si, C. Hu, and Y. Lei, “Degradation data analysis and remaining useful life estimation: a review on Wienerprocess-based methods," European Journal of Operational Research, vol. 271, no. 3, pp. 775-796, 2018.

[13] H. Wang, W. Song, E. Zio, A. Kudreyko, and Y. Zhang, "Remaining useful life prediction for lithium-ion batteries using fractional Brownian motion and fruit-fly optimization algorithm," Measurement, vol. 161, Article ID 107904, 2020.

[14] L. Bian and N. Gebraeel, "Stochastic modeling and real-time prognostics for multi-component systems with degradation rate interactions," IIE Transactions, vol. 46, no. 5, pp. 470-482, 2014.

[15] A. H. Elwany and N. Z. Gebraeel, "Sensor-driven prognostic models for equipment replacement and spare parts inventory," IIE Transactions, vol. 40, no. 7, pp. 629-639, 2008. 
[16] J.-L. Zhang and J. Chen, "Bayesian solution to pricing and inventory control under unknown demand distribution," Operations Research Letters, vol. 34, no. 5, pp. 517-524, 2006.

[17] X. Zhang and J. Zeng, "Joint optimization of condition-based opportunistic maintenance and spare parts provisioning policy in multiunit systems," European Journal of Operational Research, vol. 262, no. 2, pp. 479-498, 2017.

[18] J. Lee, M. Ghaffari, and S. Elmeligy, "Self-maintenance and engineering immune systems: towards smarter machines and manufacturing systems," Annual Reviews in Control, vol. 35, no. 1, pp. 111-122, 2011.

[19] R. P. Covert and G. C. Philip, "An EOQ model for items with Weibull distribution deterioration," A I I E Transactions, vol. 5, no. 4, pp. 323-326, 1973.

[20] D. Petrovic, R. Roy, and R. Petrovic, "Supply chain modelling using fuzzy sets," International Journal of Production Economics, vol. 59, no. 1-3, pp. 443-453, 1999.

[21] Q. Hu, J. E. Boylan, H. Chen, and A. Labib, "OR in spare parts management: a review," European Journal of Operational Research, vol. 266, no. 2, pp. 395-414, 2018.

[22] C. C. Sherbrooke, "Metric: a multi-echelon technique for recoverable item control," Operations Research, vol. 16, no. 1, pp. 122-141, 1968.

[23] C. C. Sherbrooke, "VARI-METRIC: improved approximations for multi-indenture, multi-echelon availability models," Operations Research, vol. 34, no. 2, pp. 311-319, 1986.

[24] S. C. Graves, "A multi-echelon inventory model for a repairable item with one-for-one replenishment," Management Science, vol. 31, no. 10, pp. 1247-1256, 1985.

[25] D. Acharya, G. Nagabhushanam, and S. S. Alam, "Jointly optimal block-replacement and spare provisioning policy," IEEE Transactions on Reliability, vol. 35, no. 4, pp. 447-451, 1986.

[26] A. B. M. Zohrul Kabir and A. S. Al-Olayan, "A stocking policy for spare part provisioning under age based preventive replacement," European Journal of Operational Research, vol. 90, no. 1, pp. 171-181, 1996.

[27] A. Brezavscek and A. Hudoklin, "Joint optimization of blockreplacement and periodic-review spare-provisioning policy," IEEE Transactions on Reliability, vol. 52, no. 1, pp. 112-117, 2003.

[28] T. S. Vaughan, "Failure replacement and preventive maintenance spare parts ordering policy," European Journal of Operational Research, vol. 161, no. 1, pp. 183-190, 2005.

[29] A. Van Horenbeek, J. Buré, D. Cattrysse, L. Pintelon, and P. Vansteenwegen, "Joint maintenance and inventory optimization systems: a review," International Journal of Production Economics, vol. 143, no. 2, pp. 499-508, 2013.

[30] Z. Wang, W. Wang, C. Hu, and X. Liu, "An integrated decision model for critical component spare parts ordering and condition based replacement with prognostic information," Chemical Engineering Transactions, vol. 33, pp. 1063-1068, 2013.

[31] Z.-Q. Wang, W. Wang, C.-H. Hu, X.-S. Si, and W. Zhang, “A prognostic-information-based order-replacement policy for a non-repairable critical system in service," IEEE Transactions on Reliability, vol. 64, no. 2, pp. 721-735, 2015.

[32] F. Zhao, F. Xie, C. Shi, and J. Kang, "A joint inspection-based preventive maintenance and spare ordering optimization policy using a three-stage failure process," Complexity, vol. 2017, Article ID 8319485, 19 pages, 2017.

[33] M. Zheng, H. Ye, D. Wang, and E. Pan, "Joint optimization of condition-based maintenance and spare parts orders for multi-unit systems with dual sourcing," Reliability Engineering \& System Safety, vol. 210, Article ID 107512, 2021.
[34] L. Wang, J. Chu, and W. Mao, “An optimum condition-based replacement and spare provisioning policy based on Markov chains," Journal of Quality in Maintenance Engineering, vol. 14 , no. 4 , pp. 387-401, 2008.

[35] L. Wang, J. Chu, and W. Mao, "A condition-based replacement and spare provisioning policy for deteriorating systems with uncertain deterioration to failure," European Journal of Operational Research, vol. 194, no. 1, pp. 184-205, 2009.

[36] W. Zhou, D. Wang, J. Sheng, and B. Guo, "Collaborative optimization of maintenance and spare ordering of continuously degrading systems," Journal of Systems Engineering and Electronics, vol. 23, no. 1, pp. 63-70, 2012.

[37] W. J. Kennedy, J. Wayne Patterson, and L. D. Fredendall, "An overview of recent literature on spare parts inventories," International Journal of Production Economics, vol. 76, no. 2, pp. 201-215, 2020.

[38] V. V. Kalašnikov, Mathematical Methods in Queuing Theory, Kluwer, Dordrecht, Netherlands, 2010.

[39] M. Issa, A. E. Hassanien, D. Oliva et al., "ASCA-PSO: adaptive sine cosine optimization algorithm integrated with particle swarm for pairwise local sequence alignment," Expert Systems with Applications, vol. 99, pp. 56-70, 2018.

[40] Y. Wang and Q. Shi, "Improved dynamic PSO-based algorithm for critical spare parts supply optimization under (T, S) inventory policy," IEEE Access, vol. 7, pp. 153694-153709, 2019.

[41] W.-F. Leong and G. G. Yen, "PSO-based multiobjective optimization with dynamic population size and adaptive local archives," IEEE Transactions on Systems, Man, and Cybernetics, Part B (Cybernetics), vol. 38, no. 5, pp. 1270-1293, 2008.

[42] M. J. Zuo, R. Renyan Jiang, and R. C. M. Yam, "Approaches for reliability modeling of continuous-state devices," IEEE Transactions on Reliability, vol. 48, no. 1, pp. 9-18, 1999.

[43] L. Xiao, "Joint Optimization of Spare Parts Ordering and Maintenance Policies for System with Multiple Identical Parts, Nanjing University of Aeronautics and Astronautics, Nanjing, China, 2016.

[44] X. Zhang, L. Wang, and F. Zhao, "Optimization of a joint maintenance and spare ordering policy based on remaining useful life prediction," Industrial Engineering Journal, vol. 23, no. 4, pp. 106-113, 2020. 